\title{
Sediment transport via dam-break flows over sloping erodible beds
}

\author{
M. Emmett \& T. B. Moodie \\ University of Alberta, Canada
}

\begin{abstract}
When a semi-infinite body of homogeneous fluid initially at rest behind a vertical retaining wall is suddenly released by the removal of the barrier, the resulting flow over a horizontal or sloping bed is referred to as a dam-break flow. When bed resistance is neglected the exact solution, in the case of a stable horizontal bed, may be obtained on the basis of shallow-water theory via the method of characteristics and the results are well known. Discrepancies between these shallow-water based solutions and experiments have been partially accounted for by the introduction of flow resistance in the form of basal friction. This added friction significantly modifies the wave speed and flow profile near the head of the wave so that the simple exact solutions no longer apply. Various asymptotic or numerical approaches must be implemented to solve these frictionally modified depth-averaged shallow-water equations. When the bed is no longer stable so that solid particles may be exchanged between the bed and the fluid, the dynamics of the flow become highly complex as the buoyancy forces vary in space and time according to the competing rates of erosion and deposition. It is our intention here to study dam-break flows over erodible sloping beds as agents of sediment transport, taking into account basal friction as well as the effects of particle concentrations on flow dynamics including both erosion and deposition. We shall consider shallow flows over initially dry beds and investigate the effects of changes in the depositional and erosional models employed, in the nature of the drag acting on the flow, and in the slope of the bed. These models include effects hitherto neglected in previous studies and offer insights into the transport of sediment in the worst case scenario of the complete and instantaneous collapse of a dam. Keywords: gravity current, dam-breakflow, sediment transport, dilute sediment.
\end{abstract}




\section{Introduction}

Dam-break flows, which are represented by the sudden release of fluid contained in a semi-infinite reservoir behind a vertical barrier, are of both practical and fundamental importance in fluid mechanics, engineering, and geomorphology. They have played a crucial role in underpinning simple models for a number of natural and catastrophic events, such as break-out floods from the failure of end moraine dams and various sheet flow events, as well as the formative stages of lahars or debris flows [1-3]. Although in practice the release of water upon collapse of the retaining barrier will often be more gradual than that in the idealised mathematical models, one can view these models as providing the worst case scenario for these events $[3,4]$.

The earliest work on dam-break flows considered single phase, low aspect ratio, frictionless flows in rectangular geometry taking the shallow-water equations as the governing model equations. With the bed below the dam assumed horizontal and dry, the solution for the flow is a centred simple wave that was first developed by Ritter [5]. If the initial depth of water behind the vertical dam is $h_{0}$, the front of the flow advances as a wave over the dry bed with constant speed $2 \sqrt{g h_{0}}$, while the reduction of depth spreads back from the initial position of the dam with speed $\sqrt{g h_{0}}$, where $g$ is the acceleration due to gravity. In the disturbed region between the two extremes of depth, the velocity $u$ and the depth of the flow $h$ are given by

$$
\begin{gathered}
u=\frac{2}{3}\left(\frac{x}{t}+\sqrt{g h_{0}}\right), \quad \text { and } \\
\sqrt{g h}=\frac{1}{3}\left(2 \sqrt{g h_{0}}-\frac{x}{t}\right),
\end{gathered}
$$

where $x$ measures distance downstream of the original position of the dam and $t$ measures the time elapsed since its collapse. Although these solutions do provide a reasonably good match to the experimental observations when the transients associated with the initial release have died down there are still important properties of the flow that are not captured by the classical shallow-water model used in the construction of the solutions displayed in (1) and (2). It has been observed in particular $[3,6]$ that for the dam-break flow experiments the water near the tip piles up and the front speed is appreciably less than that predicted by the simple theory.

In order to account for this blunting of the tip and the slowing down of the front several authors $[4,7,8]$ have postulated that near the tip, where the depth of flow drops to zero, frictional resistance and the resulting turbulence dominate the flow. To account for this basal friction a Chézy resistance term is added to the momentum equation $[4,8]$. Various asymptotic procedures were employed $[4,7,8]$ to determine the influence of this resistance and it was found that its inclusion brought theory and experiment into closer accord. Since we are interested in developing and exploiting models for sediment transport that employ dambreak flows on down-sloping topography as paradigms for certain geological and engineering processes we shall extend the model beyond what is discussed in this 
paragraph while appreciating the significant gains achieved through the addition of the resistance term. In fact, basal friction with realistic parameterisations for geological applications appears to have a much greater influence on flow dynamics than does the presence of particles in suspension [9].

Recent studies [1,2] have employed dam-break flows as agents of sediment transport. In [2] the authors explore dam-break flows over beds that consist of fine sediment that can be entrained into the water column and transported in suspension. The sediment transport was passive in that the suspended particles did not influence the flow dynamics which could then be totally specified employing the simple exact solution of the shallow-water equations for both a dry bed [5] and a bed with 'tail water'. Recent studies by the current authors $[9,10]$ have shown that under the assumption of dilute suspensions employed in [2] the suspended particles will play a relatively minor role in modifying the flow dynamics so that passivity of sediment transport does not produce large errors. The omissions of basal friction and bed topography however, as was the case in [2], were shown to have a more profound negative influence on the accuracy of the results [10].

In the present work we shall employ dam-break flows over erodible beds as agents of sediment transport. The inclusion of a velocity dependent basal friction as well as bottom topography and non-passive particle transport adds several important mechanisms that were absent from previous studies. We shall assume that our flows are shallow so that the pressure remains hydrostatic throughout the flow regime [2,8-13]. We shall also assume that the particle concentration in the flow remains sufficiently low so that we may treat the particles as being isolated and employ a Boussinesq approximation whereby the particles appear in the momentum equations only in the buoyancy terms. These assumptions put definite constraints on the range of particle volume fractions $\phi(x, t)$ in our well-mixed suspensions. When erosion exceeds deposition so that particle concentrations are increasing we shall assume that our model calculations are valid up until $\phi \approx 0.05$ $[9,10]$. Although the bottom boundary shear stress could be calculated from the full governing equations we shall adopt the common and much simpler approach of introducing a Chézy drag coefficient $C_{D}$ which when viscous effects are small (large Reynolds number flows) gives the boundary shear stress as $\tau_{b}=C_{D} \rho_{f} u^{2}$, where $\rho_{f}$ is the density of the fluid and $u$ a depth averaged horizontal velocity [4,8-10]. The Chézy drag coefficient is dimensionless and usually falls in the range 0.01-0.001 for most environmental flows [8]. With all of our additions to the model of Pritchard and Hogg [2] (basal drag, bottom topography, and particle modified flow dynamics) the simple shallow-water based solutions [5] will no longer apply and the approach adopted in [2] is not available so that an alternative approach will have to be adopted.

\section{Model development}

We consider the two-dimensional flow resulting from the sudden release of water initially held at rest behind a plane vertical retaining wall of height $h_{0}$. The bed below the dam which is initially located at $x=0$, is gently varying and specified 


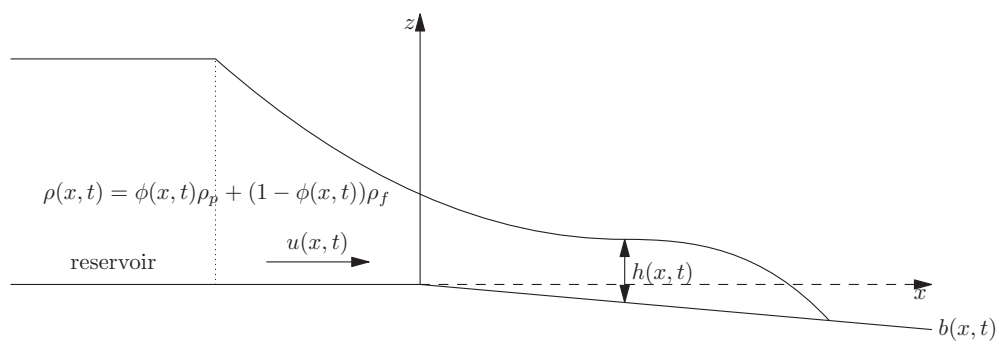

Figure 1: Schematic for the dam-break flow on a dry sloping and erodible bed.

by the topography $z=b(x, t)$. It is assumed that initially there is no water below the dam and that the bed is comprised of fine or cohesive material which, once a threshold shear stress is exceeded, is entrained into the water column and transported in suspension to possibly be deposited downstream. With our assumption of dilute suspensions the erosion or deposition depth is small relative to the depth of the current so that the position of the bottom boundary may be kept fixed in most of our calculations. Although in the early stages of such dam-break flows the stream-wise and vertical scales of the motion will be comparable, there soon comes a time when stream-wise scales dominate the vertical ones and the flow may be considered to be a shallow flow with negligible vertical accelerations and a hydrostatic pressure distribution can be adopted [2,8].

We shall assume that once particles have been suspended into the water column they are always vertically well-mixed by the turbulence of the flow $[2,11,12,14]$ so that the volume fraction of particles in suspension $\phi$ is a function of the horizontal coordinate $x$ and the time $t$ elapsed since collapse of the dam. We shall always assume that the water initially behind the dam is particle free. The bulk density $\rho$ of the suspension is then given by

$$
\rho(\phi)=\rho_{p} \phi+(1-\phi) \rho_{f}
$$

where $\rho_{p}\left(>\rho_{f}\right)$ is the particle density. The setting under which the model is developed is summarised by the schematic presented in Figure 1.

The depth-averaged continuity and momentum equations for the system under the Boussinesq approximation $(\phi \ll 1)$ and the hydrostatic assumption [10] are

$$
\begin{gathered}
\frac{\partial h}{\partial t}+\frac{\partial}{\partial x}(h u)=0, \quad \text { and } \\
\frac{\partial}{\partial t}(h u)+\frac{\partial}{\partial x}\left(\beta h u^{2}+\frac{1}{2} \frac{g}{\rho_{f}} \rho h^{2}\right)=-\frac{\rho g h}{\rho_{f}} \frac{\partial b}{\partial x}-C_{D} u^{2},
\end{gathered}
$$

where $\beta$ is the Boussinesq coefficient (shape factor) [8]. The magnitude of $\beta \geq 1$ corresponds to the amount of shear present in the horizontal velocity field and may depend on such factors as the Reynolds number or the boundary roughness [8]. The 
particle mass conservation and bed evolution equations $[9,10,12]$ are

$$
\frac{\partial}{\partial t}\left(\rho_{p} \phi h\right)+\frac{\partial}{\partial x}\left(\rho_{p} \phi h u\right)=q_{e}-q_{d}, \quad \text { and } \rho_{p} \frac{\partial b}{\partial t}=-q_{e}+q_{d} .
$$

where $q_{e}$ is the mass erosion flux and $q_{d}$ is the mass deposition flux.

Observations have shown that $q_{e}$ and $q_{d}$ are functions of both the fluid velocity $u$ and the volume fraction of particles in suspension $\phi$ [15]. We adopt the usual expression for mass deposition rate, that is, $q_{d}=\rho_{p} \phi v_{s}$, where $v_{s}$ is the Stokes settling velocity [2,9-14]. With no particles in suspension behind the dam we need only consider deposition when $u>u_{c}$, where $u_{c}$ is some critical velocity below which particles are not entrained into the fluid column. We adopt the expression

$$
q_{e}(u)= \begin{cases}\rho_{p} v_{e}\left(\frac{u^{2}}{u_{c}^{2}}-1\right)^{n} & \text { for }|u| \geq u_{c}, \\ 0 & \text { for }|u|<u_{c}\end{cases}
$$

for the erosion rate $q_{e}$, where $v_{e}$ is a sediment entrainment rate. This model is used to describe the erosion of sediment from a cohesive bed or from a bed of fine cohesionless material where some critical shear stress must be exceeded in order to entrain particles from the bed into the water column [2,9,10,15-17].

We make all equations non-dimensional using the non-dimensionalisation and scaling scheme presented in [10]. Of particular importance is the typical volume fraction scale $\phi_{0}$ and velocity scale $U$. The velocity scale $U$ is the familiar $\sqrt{\hat{g} h_{0}}$ wherein $\widehat{g}$ is a 'modified gravity' which is given by $\left(\gamma \phi_{0}+1\right) g$ where $\gamma=\left(\rho_{p}-\rho_{f}\right) / \rho_{f}$. Rendering all equations non-dimensional gives

$$
\begin{aligned}
& \frac{\partial}{\partial t} h+\frac{\partial}{\partial x}(h u)=0, \\
& \frac{\partial}{\partial t}(h u)+\frac{\partial}{\partial x}\left[\beta h u^{2}+\frac{1}{2} h^{2}(1-\Gamma+\phi \Gamma)\right]=-h(1+\phi \Gamma) b_{x}-C_{D} u^{2}, \\
& \frac{\partial}{\partial t}(\phi h)+\frac{\partial}{\partial x}(\phi h u)=q_{e}-q_{d}, \\
& \frac{\partial b}{\partial t}=\phi_{0}\left(q_{d}-q_{e}\right), \\
& q_{d}=u_{d} \phi, \quad \text { and } \quad q_{e}(u)= \begin{cases}u_{e}\left(\frac{u^{2}}{u_{c}^{2}}-1\right)^{n} & \text { for }|u| \geq u_{c}, \\
0 & \text { for }|u|<u_{c}\end{cases}
\end{aligned}
$$

where we have introduced the non-dimensional parameter $\Gamma=1-1 /\left(\gamma \phi_{0}+1\right)$.

\section{Analysis of the model}

In this section we highlight various aspects of dam-break flows over erodible beds based upon our model equations. The parameter values used in the simulations are typical for flows of a geological scale with $h_{0}=20 \mathrm{~m} \mathrm{[11].}$ 
(a) $h(x, t)$ vs $x$

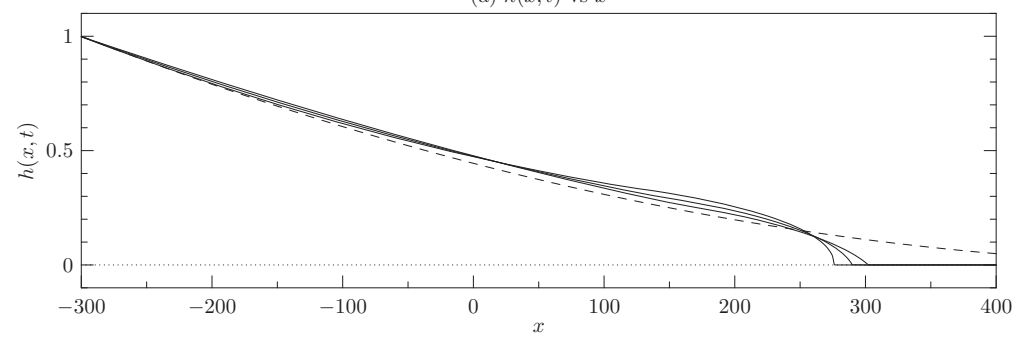

(b) $u(x, t)$ vs $x$

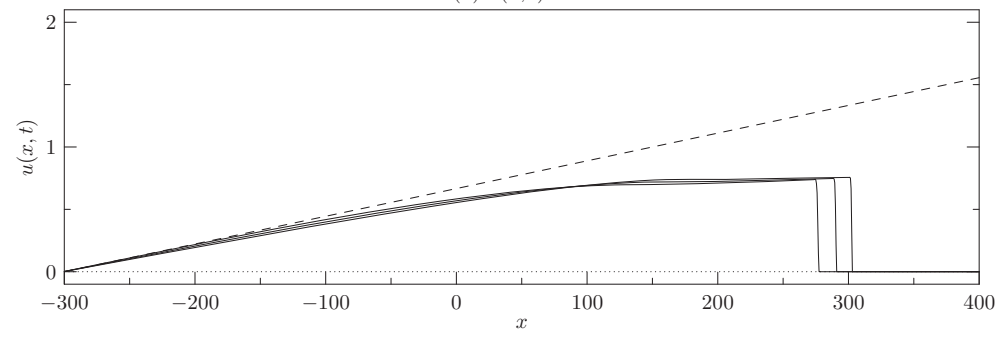

Figure 2: Solutions of (a) height and (b) velocity of the particle free modified dambreak flow over a flat bottom with basal drag at $t=300$. Solid lines show numerical solutions with $\beta=1.0,1.1$, and 1.2 (from left to right, at the front). Dashed line shows the Ritter solution. Parameter value used is $C_{D}=0.001$.

We begin by presenting an exploration of the effects of those mechanisms (velocity shear, bed slope, and drag) that do not necessarily involve suspended particles. To do so we examine solutions of the initial value problem corresponding to the dam-break flow for the non-dimensional pair of coupled equations (8) and (9) with $\Gamma \equiv 0$. Subsequently, we present an overview of the effects of suspended particles by allowing the flows to entrain particles through bed erosion but fixing the bed so that $b(x, t)$ is in fact independent of $t$. A more detailed study of these scenarios is presented in [10]. Finally, we present a brief note regarding the evolution of the bed topography when the bed is eroded according to (11).

\subsection{Modified dam-break flows with drag}

In this subsection we present solutions for modified dam-break flows with basal drag in the absence of bed topography or particles. That is, following the approach employed in $[2,6-9,18]$ we retain the basal drag term in order to bring our model calculations into closer accord with the experimental results of [7] wherein dambreak flows over a horizontal bed were examined.

In Figure 2 we have plotted both the height and velocity profiles, respectively, for both the classic solution [5] and the numerical solution including basal drag. 
We note immediately that the presence of drag has significantly altered the shape of the depth profile in the immediate vicinity of the leading edge as well as the velocity structure of the flow in this region. We also note that, in agreement with the experimental results [7] and the theoretical approach adopted in [4], the velocity is nearly uniform in the blunt snout. That is, in the deformed tip basal drag retards the flow so that the velocity profile is approximately horizontal there. The assumption that the velocity in the tip depends only on time was crucial to the theoretical development in [4] and appears to be confirmed by our numerical work.

Furthermore, we note that the effect of vertical shear $(\beta>1)$ in the horizontal velocity profile is most dramatic in the immediate vicinity of the leading edge where the depth, and hence momentum, of the flow is small allowing the effect of vertical shear in the horizontal velocity to be accentuated.

\subsection{Modified dam-break flows with drag over a linear slope}

In this subsection we will examine modified dam-break flows over sloping beds in order to isolate the effects of the interplay between the bottom slope and basal drag. We shall take the bottom topography to be specified by

$$
b(x, t)=-s x \Theta(x)
$$

where $s$ is a small non-dimensional parameter and $\Theta$ is the Heaviside step function. Employing this simple linear form for the bottom topography allows us to appeal to our intuition while interpreting both theoretical and numerical results. Furthermore, the stream-wise gradient of a linear slope is constant, affording us the opportunity to perform an asymptotic expansion over the bed slope $s$, which was presented in [10].

In Figure 3 we have plotted the numerical solutions for both the height and velocity profiles of a particle free dam-break flow over a sloping bottom with drag for $\beta=1$. We note that both the height and velocity profiles are nearly horizontal in the bulk of the flow over the linearly sloping bed. In the presence of a sloping bottom the blunt snout in the height profile has become more abrupt and falls steeply to zero at the front. As demonstrated in previous sections, the effect of drag is to retard the front and create a blunt snout, while the effect of a sloping bottom is to draw out the fluid, reducing its height in the bulk of the flow over the sloping bed, and slightly increasing its height directly behind the front. Furthermore, in contrast to the drag-free case, the presence of a sloping bottom has a significant effect on the front position of the flow. As one may expect, the front position is greater for flows over steeper beds.

\subsection{Modified dam-break flows with drag and sediment}

In this subsection we will examine modified dam-break flows as agents of sediment transport over flat erodible beds while allowing the particle volume fraction to change through the mechanisms of particle advection, deposition, and entrainment through bed erosion. In our previous work [9] we demonstrated the role played 
(a) $h(x, t)$ vs $x$

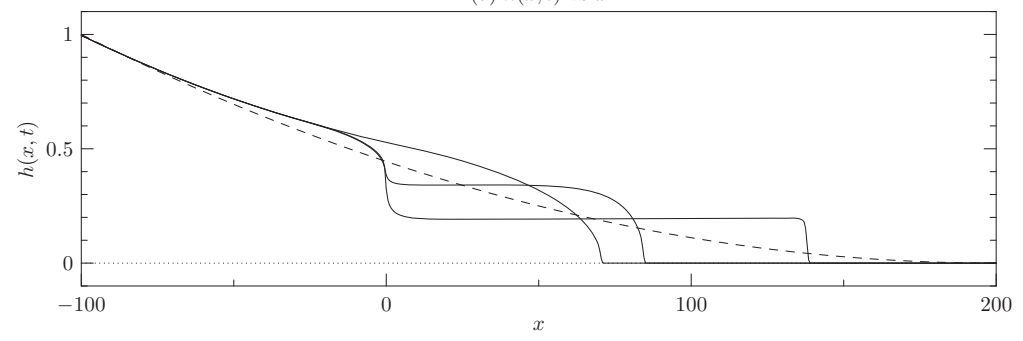

(b) $u(x, t)$ vs $x$

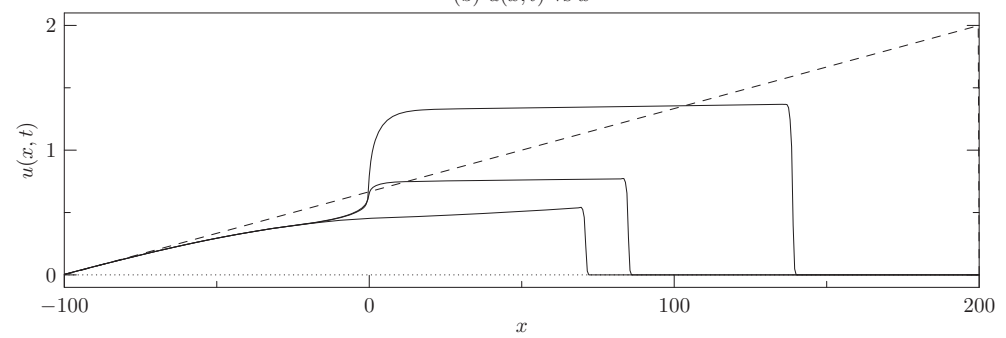

Figure 3: Solutions of (a) height and (b) horizontal velocity for particle free dambreak flows over linear slopes with drag. Solid lines show numerical solutions at $t=100$ with slope $s=0,0.001$, and 0.01 (from left to right, at the front). Dashed lines show the Ritter solutions. Parameter values used are $C_{D}=0.01$ and $\beta=1$.

by the critical bed velocity $u_{c}$ in the long term competition between erosion and deposition and displayed the strong effect of basal drag on the ultimate outcome of this competition. Furthermore, we observed that the inclusion of particles did not have a significant effect on the height or velocity profiles of the flows. Generally, flows with particles were slightly faster compared to analogous particle free flows, but only by a few percent at most.

The maximum rate of sediment entrainment occurred at the front and was nearly uniform within the snout. Peaks in the volume fraction of sediment profiles were observed directly behind the front where the height of the fluid decreased sharply to zero. These peaks in the volume fraction of sediment profiles where highest for short post-release times since the velocity was also highest for short post-release times, and decreased with time.

In Figure 4 we have plotted a typical numerical solution of the full model equations with drag, particle deposition, and bed erosion over a flat bed for a parameter configuration in which the drag coefficient $C_{D}$ and critical bed velocity $u_{c}$ were small enough so that particles were entrained by the snout for the duration of the flow.

The interplay between basal drag and the critical bed velocity was further demonstrated in our recent work [9] by examining the particle flux at a fixed 


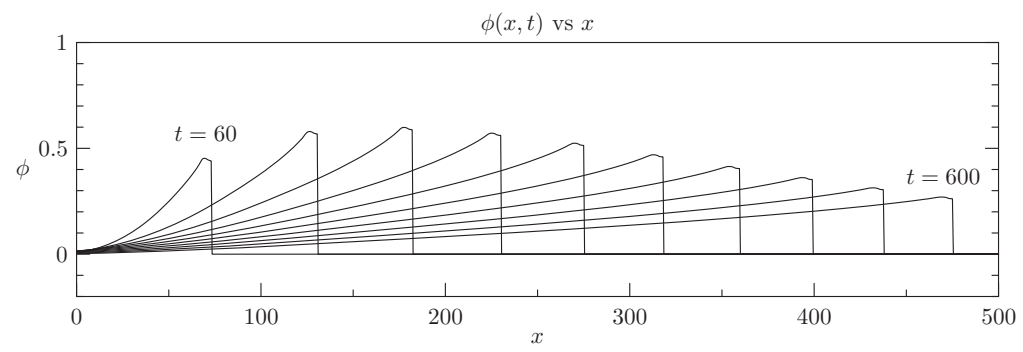

Figure 4: Solutions (volume fraction) of the dam-break flow over a flat bed with basal drag, particle deposition, and particle entrainment at various times $t=60,120, \ldots, 540,600$. Parameter values used are $C_{D}=0.001$, $\phi_{0}=0.01, \gamma=2.5, u_{d}=0.005, u_{e}=0.0015, n=1.2, u_{c}=0.5$, and $\beta=1$.

station. Flows with high basal drag did not continue to erode the bed and entrain particles once the front had passed a given station. Flows with a critical bed velocity above $2 / 3$ were also dominated by deposition since the long term Ritter velocity is less than $2 / 3$ regardless of the presence of basal drag. Flows with low drag and a critical bed velocity below $2 / 3$ continued to erode the bed and advect entrained particles downstream. As time progressed, the horizontal velocity at the station approached its Ritter solution and the particle flux due to erosion approached a steady value while the particle flux due to deposition increased with increasing volume fraction until an equilibrium between erosion and deposition was reached.

\subsection{Modified dam-break flows with drag and sediment over a linear slope}

In this subsection we will examine modified dam-break flows as agents of sediment transport over sloping erodible beds.

In our previous work [10] we observed that, as in the previous case with flat beds, the inclusion of particles did not have a significant effect on the height or velocity profiles of the flows. Particles entrained into the flow maintained their relative position within the flow which resulted in a nearly linear volume fraction profile that increased in the downstream direction. Furthermore, the maximum attained by the volume fraction continued to increase for all post-release times, in contrast to the flat bed case. The peak in the volume fraction occurred directly behind the front, and was primarily due to advection coupled with the nearly horizontal velocity profile over the sloping bed.

In Figure 5 we have plotted the horizontal velocity $u$ and volume fraction of sediment $\phi$ for a typical numerical solution to the full model equations with basal drag, particle deposition, bed erosion, and bed slope. These plots elucidate the relationship between the velocity and volume fraction of sediment entrained by the flow. 

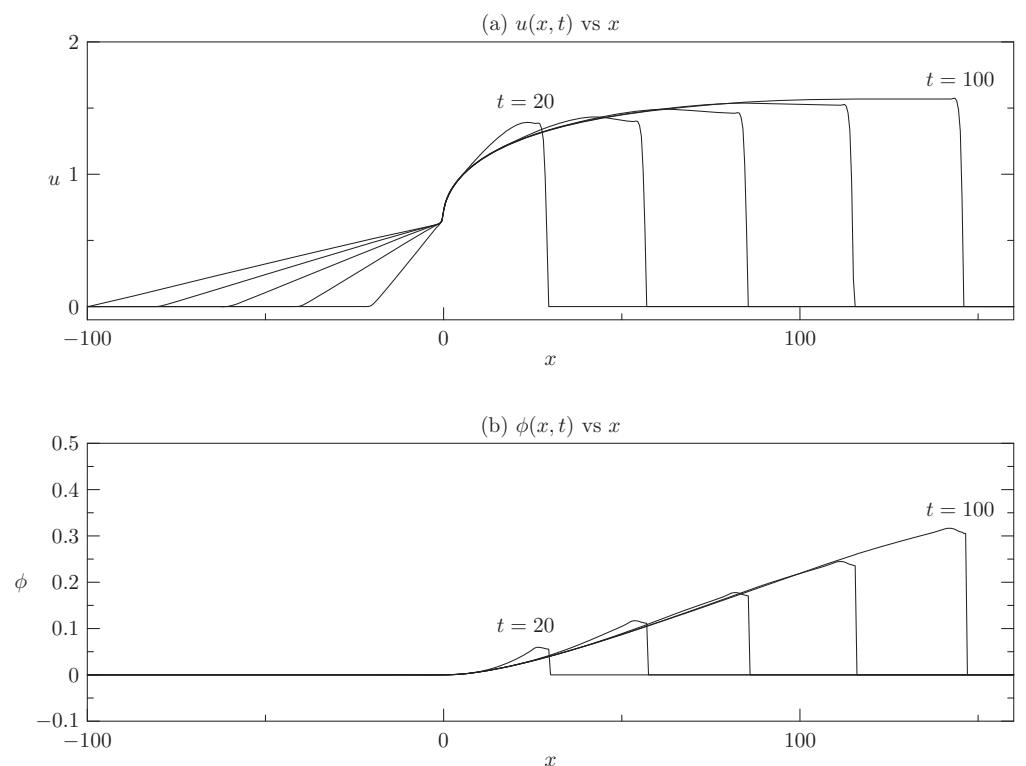

Figure 5: Solutions (a) horizontal velocity and (b) volume fraction of the dambreak flow with basal drag, particle deposition, and bed erosion at various times $t=20,40, \ldots, 80,100$. Parameter values used are $C_{D}=0.001$, $\phi_{0}=0.01, \gamma=2.5, u_{d}=0.005, u_{e}=0.0015, n=1.2, u_{c}=0.5$, $\beta=1$, and $s=0.001$.

The linear nature of the volume fraction profile shown in Figure 5 is in contrast to the flat case (see Figure 4) in which the volume fraction was highest in the snout and decayed in a non-linear fashion in the upstream direction [9]. In the sloping case, the peak in the volume fraction is primarily due to advection coupled with the nearly horizontal velocity profile, and the peak increases for all post-release times.

\subsection{Modified dam-break flows with drag and sediment over a variable bed}

In this subsection we will examine modified dam-break flows as agents of sediment transport over initially flat erodible beds. We allow the bed topography to change with time through the mechanism of erosion according to (11).

In Figure 6 we have plotted the bed topography $b(x, t)$ and horizontal velocity $u$ of the numerical solution to the full model equations with basal drag, particle deposition, and bed erosion. These plots show the scour pit resulting from bed erosion. We note that the pit is deepest slightly downstream from the original position of the dam. There is peak in the horizontal velocity directly above the scour pit since the fluid gains momentum as it falls into the pit. 


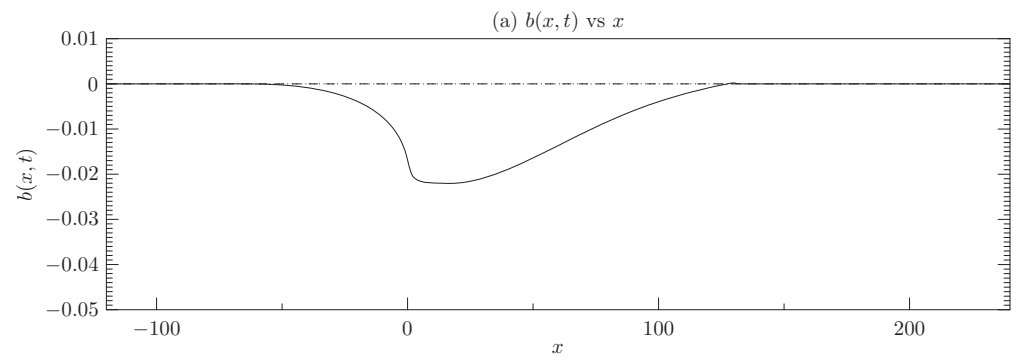

(b) $u(x, t)$ vs $x$

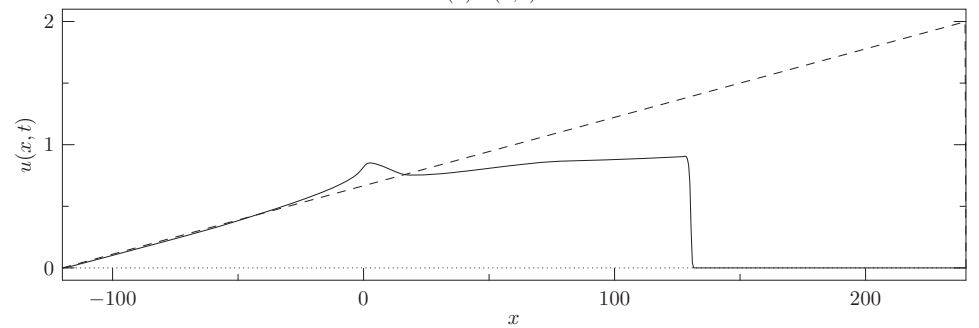

Figure 6: Solutions of (a) bed height and (b) horizontal velocity for particle laden dam-break flows over variable beds with drag at time $t=120$. Solid lines show numerical solutions with $C_{D}=0.001, \phi_{0}=0.01, \gamma=2.5$, $u_{d}=0.005, u_{e}=0.0015, n=1.2, u_{c}=0.5, \beta=1$, and $s=0.001$. Dashed lines show the Ritter solutions.

Further exploration of the complex interplay between the fluid, sediment, and bed dynamics will be carried out in subsequent research.

\section{Discussion}

We have developed a model to describe the transport of dilute sediment under dam-break flows over sloping beds with basal drag and presented numerical solutions to this model in order to investigate the influence of various model parameters. The model developed is an extension to previous models, includes a velocity dependent basal drag force, incorporates the effects of a spatially dependent bed topography, and allows the variable concentration of suspended particles, through the mechanisms of deposition and erosion, to influence the flow dynamics. The model is unique from existing models which do not couple the flow and sediment dynamics and do not include basal drag [2] or bed topography [9]. The numerical results show that this coupling is especially important for the sediment dynamics and should not be ignored when the understanding of sediment processes is vital to a given study. 


\section{References}

[1] Capart, H. \& Young, D., Formation of a jump by the dam-break wave over a granular bed. J Fluid Mech, 372, pp. 165-187, 1998.

[2] Pritchard, D. \& Hogg, A., On sediment transport under dam-break flow. $J$. Fluid Mech, 473, pp. 265-274, 2002.

[3] Stansby, P., Chegini, A. \& Barnes, T., The initial stages of dam-break flow. J. Fluid Mech, 374, pp. 407-424, 1998.

[4] Whitham, G., The effects of hydraulic resistance in the dambreak problem. Proc R Soc London, Ser A, 227, pp. 399-407, 1955.

[5] Ritter, A., Die fortpflanzung der wasserwellen. Z Verein Deutsch Ing, 36, pp. 947-954, 1892.

[6] Dressler, R., Hydraulic resistance effect upon the dam-break functions. J. Res Nat Bur Stand, 49, pp. 217-225, 1952.

[7] Dressler, R., Comparison of theories and experiments for the hydraulic dambreak wave. Proceedings of the Commission des Eaux de Surface at the Assemble Gnrale de Rome 1954, International Association of Hydrological Sciences, volume 38, pp. 319-328, 1954.

[8] Hogg, A. \& Pritchard, D., The effects of hydraulic resistance on dam-break and other shallow inertial flows. J Fluid Mech, 501, pp. 179-212, 2004.

[9] Emmett, M. \& Moodie, T., Dam-break flows with resistance as agents of sediment transport. Phys Fluids, 20(8), p. 086603, 2008.

[10] Emmett, M. \& Moodie, T., Sediment transport via dam-break flows over sloping erodible beds. Stud Appl Math, 2009. Submitted.

[11] Bonnecaze, R., Huppert, H. \& Lister, J., Particle-driven gravity currents. $J$. Fluid Mech, 250, pp. 339-369, 1993.

[12] Moodie, T., Pascal, J. \& Swaters, G., Sediment transport and deposition from a two-layer fluid model of gravity currents on sloping bottoms. Stud Appl Math, 100, pp. 215-244, 1998.

[13] Moodie, T. \& Pascal, J., Axisymmetric particle-bearing gravity flows on sloping bottoms. Can Appl Math Q, 7, pp. 17-47, 1999.

[14] Moodie, T. \& Pascal, J., Non-hydraulic effects in particle-driven gravity currents in deep surroundings. Stud Appl Math, 107, pp. 217-251, 2001.

[15] Teisson, C., Ockenden, M., Hir, P.L., Kranenburg, C. \& Hamm, L., Cohesive sediment transport processes. Coastal Eng, 21, pp. 129-162, 1993.

[16] Kerswell, R.R., Dam break with Coulomb friction: a model for granular slumping? Phys Fluids, 17(5), pp. 057101, 16, 2005.

[17] Blanchette, F., Strauss, M., Meiburg, E., Kneller, B. \& Glinsky, M., Highresolution numerical simulations of resuspending gravity currents: Conditions for self-sustainment. J Geophys Res, 110, p. C12022, 2005.

[18] Whitham, G., Linear and Nonlinear Waves. Wiley, New York, 1974. 